\title{
STATIONARY HARMONIC FUNCTIONS ON HOMOGENEOUS SPACES
}

\author{
V.S. KHOROSHCHAK, A.A. KONDRATYUK
}

\begin{abstract}
Stationary harmonic functions on homogeneous spaces are considered. A relation to double periodic harmonic functions of three variables is showed.

Keywords: harmonic function, multiplicatively periodic function, double periodic function, homogeneous space, Klein space, invariant family, stationary element with respect to a subgroup, punctured Euclidean space.
\end{abstract}

Mathematics Subject Classification: 31B05

\section{INTRODUCTION}

A function $f$ on $\mathbb{C}^{*}=\mathbb{C} \backslash\{0\}$ is said to be multiplicatively periodic if there exists $q$, $0<|q| \neq 1$ such that

$$
\forall z \in \mathbb{C}^{*} \quad f(q z)=f(z) .
$$

Such a $q$ is called multiplicator of $f$.

The theory of meromorphic functions satisfying (1) is dual to the theory of elliptic functions, which are double periodic meromorphic functions on $\mathbb{C}([1]-[3])$.

Holomorphic functions, harmonic functions, subharmonic functions satisfying (1) are constant.

In this connection we try to answer the questions:

(i) do multiplicatively periodic non-constant harmonic functions of several variables exist?

(ii) do double periodic non-constant harmonic functions of three variables exist?

(iii) if yes, what are their representations?

Note that $\mathbb{C}^{*}$ is a nonlinear homogeneous space on which multiplicative group $\mathbb{C}^{*}$ acts and that (1) implies

$$
\forall n \in \mathbb{Z} \quad \forall z \in \mathbb{C}^{*} \quad f\left(q^{n} z\right)=f(z) .
$$

We will say that $f$ is stationary with respect to the cyclic group $\left\{q^{n}\right\}, n \in \mathbb{Z}$, generated by $q$.

Note also that each multiplicatively periodic harmonic functions of multiplicator $q, 0<q \neq 1$, in the punctured Euclidean space $\stackrel{\circ}{\mathbb{R}}^{m}=\mathbb{R}^{m} \backslash\{0\}, m \geq 3$, is constant due to the extremum principle and the counterpart of the Liouville theorem. Hence, in order to solve problems (i)-(iii) we should consider more general homogeneous spaces.

\section{Homogeneous SPACES}

Definition 1. Let $X$ be a topological space, $G$ be a group of homeomorphic mappings of $X$ onto $X$. A couple $(X, G)$ is called homogeneous space.

V.S. Khoroshchak, A. A. Kondratyuk, Stationary harmonic functions on homogeneous SPACES.

(c) Khoroshchak V.S., Kondratyuk A.A. 2015. 
If $G$ is transitive, that is

$$
\forall x_{1}, x_{2} \in X \quad \exists \tau \in G \quad\left(x_{2}=\tau x_{1}\right),
$$

then $(X, G)$ is said to be the Klein space (see [4]).

Example 1. Let $G$ be a group of the linear transformations of Euclidean space $\mathbb{R}^{n}$. It is transitive. Then $\left(\mathbb{R}^{n}, G\right)$ is the linear homogeneous space. It is the Klein space.

Example 2. Let $X=\mathbb{R}^{n} \backslash\{0\}, G=S O(n) . X$ is invariant with respect to homothetic transformations, $G$ is intransitive. Thus, instead of $G$ the compositions of rotations and homothetic transformations $G_{1}$ can be taken. Then $G_{1}$ is transitive and $\left(\mathbb{R}^{n} \backslash\{0\}, G_{1}\right)$ is the nonlinear homogeneous space. It is the Klein space, too.

\section{Invariant functional spaces on $(X, G)$. Stationary ElEments With Respect TO SUBGROUPS}

Definition 2. Let $(X, G)$ be a homogeneous space. A set (family) of functions $\mathcal{F}$ is said to be invariant if it satisfies the following condition

$$
\forall f \in \mathcal{F} \quad \forall \tau \in G \quad(f \circ \tau \in \mathcal{F}) .
$$

Definition 3. Let $(X, G)$ be a homogeneous space, $\mathcal{F}$ be an invariant family, $H$ be a subgroup of group $G$. An element $f \in \mathcal{F}$ is called stationary with respect to $H$ if

$$
\forall \tau \in H \quad(f \circ \tau=f) .
$$

The set of such elements is denoted by $\mathcal{F}_{H}$.

\section{Stationary haRMONiC FUnCtions ON HOMOGENEOUS SPACES}

The space $\overrightarrow{\mathbb{R}}^{3}=\left\{\left(x_{1}, x_{2}, x_{3}\right)=x: x_{1}^{2}+x_{2}^{2}>0\right\}$ is called pierced Euclidean space. It is nonlinear, invariant with respect to the rotations around axis $x_{3}$ and homothetic transformations. The composition of the rotations around axis $x_{3}$ and homothetic transformations forms a group, which we denote by $G$. Hence, we obtain nonlinear homogeneous space $\left(\overrightarrow{\mathbb{R}}^{3}, G\right)$. It is the Klein space.

One of the functional spaces invariant with respect to group $G$ is the linear space of harmonic in $\overrightarrow{\mathbb{R}}^{3}$ functions (see, for example, [5]). We denote it by $\mathcal{H}$.

The rotation by an angle $\alpha$ around axis $x_{3}$ is given by the following matrix

$$
A=\left(\begin{array}{ccc}
\cos \alpha & -\sin \alpha & 0 \\
\sin \alpha & \cos \alpha & 0 \\
0 & 0 & 1
\end{array}\right)
$$

Fix $q, 0<q<1$. Let $H$ be a composition of some $A$ and the cyclic group $\left\{q^{n}\right\}, n \in \mathbb{Z}$.

It is an open problem to describe stationary elements from $\mathcal{H}_{H}$ which are harmonic in $\overrightarrow{\mathbb{R}}^{3}$ functions satisfying the condition

$$
\forall x \in \overrightarrow{\mathbb{R}}^{3} \quad h\left(q^{n} A x\right)=h(x) .
$$

However, we can show that class $\mathcal{H}_{H}$ is non-trivial, i.e., it contains non-constant harmonic functions.

We consider the series

$$
\sum_{n=0}^{+\infty}\left(\frac{1}{|a|}-\frac{1}{\left|q^{n} x-a\right|}\right)-\sum_{n=1}^{+\infty} \frac{1}{\left|q^{-n} x-a\right|}
$$


introduced in ([6]). It was proved there that for any fixed $a \in \stackrel{\circ}{\mathbb{R}}^{3}, q<|a| \leqslant 1$, the remainder of series 22 converges uniformly on the compact subsets from $\stackrel{\circ}{\mathbb{R}}^{3}$ and that the sum $K(x, a)$ of (2) is subharmonic in $\stackrel{\circ}{\mathbb{R}}^{3}$.

Denote $X_{3}=\left\{\left(0,0, x_{3}\right): x_{3} \neq 0\right\}$. If $a \in X_{3}$, then each term of (2) is a harmonic function in $\overrightarrow{\mathbb{R}}^{3}$. Indeed, the fundamental solution of the Laplace equation in $\mathbb{R}^{3}$ is $\frac{1}{|x|}$. Since $q^{k} x \neq a$, $k \in \mathbb{Z}$, the function $\frac{1}{\left|q^{k} x-a\right|}$ is harmonic in $\overrightarrow{\mathbb{R}}^{3}$. Therefore, the function $K(x, a)$ is harmonic in $\overrightarrow{\mathbb{R}}^{3}$ if $a=\left(0,0, a_{3}\right) \in X_{3}, q<|a| \leqslant 1$.

Note that the function $K(x, a)$ is independent of $\alpha$, namely,

$$
\forall A \quad K(A x, a)=K(x, a) .
$$

Let $y=A x$, that is $y_{1}=x_{1} \cos \alpha-x_{2} \sin \alpha, y_{2}=x_{1} \sin \alpha+x_{2} \cos \alpha, y_{3}=x_{3}$. Consider the absolute value $\left|q^{k} y-a\right|, k \in \mathbb{Z}$. We have

$$
\begin{gathered}
\left|q^{k} y-a\right|=\left|\left(q^{k} x_{1} \cos \alpha-q^{k} x_{2} \sin \alpha, q^{k} x_{1} \sin \alpha+q^{k} x_{2} \cos \alpha, q^{k} x_{3}-a_{3}\right)\right|= \\
=\sqrt{q^{2 k} x_{1}^{2}+q^{2 k} x_{2}^{2}+\left(q^{k} x_{3}-a_{3}\right)^{2}}=\left|q^{k} x-a\right| .
\end{gathered}
$$

Thus, identity (3) is valid.

It is easy to check that

$$
K(q x, a)=K(x, a)-\frac{1}{|a|} .
$$

Let $a=(0,0,1)$ and $b=(0,0,-1)$.

The function

$$
h(x)=K(x, a)-K(x, b)
$$

is harmonic in $\overrightarrow{\mathrm{R}}^{3}$.

Using identities (3) and (4), we obtain

$$
h(q A x)=K(x, a)-\frac{1}{|a|}-K(x, b)+\frac{1}{|b|} .
$$

Since $|a|=|b|$, we get

$$
\forall x \in \overrightarrow{\mathbb{R}}^{3} \quad h(q A x)=h(x)
$$

Thus, $h \in \mathcal{H}_{H}$.

\section{A CLASS OF FUNCTIONS IN $\mathcal{H}_{H}$}

We denote by $\mathcal{B}$ the class of bounded Borel sets in $\stackrel{\circ}{\mathbb{R}}^{3}$ whose closures belong to $\stackrel{\circ}{\mathbb{R}}^{3}$. For $B \in \mathcal{B}$ we let

$$
q B=\{q x: x \in B\}, \quad 0<q<1 .
$$

Theorem A ([7]). A measure $\mu$ in $\stackrel{\circ}{\mathbb{R}}^{3}$ is the Riesz measure of a multiplicatively periodic $\delta$-subharmonic functions of multiplicator $q$ if and only if

(i) $\mu(q B)=q \mu(B)$ for each $B \in \mathcal{B}$;

(ii) $\int_{q r<|x| \leqslant r} \frac{d \mu}{|x|}=0$ for all $r>0$.

Theorem B ([7]). Each multiplicatively periodic $\delta$-subharmonic in $\stackrel{\circ}{\mathbb{R}}^{3}$ function $u$ of multiplicator $q$ satisfies the representation

$$
u(x)=C+\int_{q<|a| \leqslant 1} K(x, a) d \mu_{u}(a),
$$


where $C$ is a constant.

The following theorem describes a class of harmonic functions from $\mathcal{H}_{H}$.

Theorem 1. If a Borel measure $\mu$ on $\stackrel{\circ}{\mathbb{R}}^{3}$ satisfies the conditions

1) $\mu(q B)=q \mu(B)$ for each $B \in \mathcal{B}$;

2) $\int_{q<|a| \leqslant 1} \frac{d \mu}{|a|}=0$;

3) $\mu(B)=\mu\left(B \bigcap X_{3}\right), \quad \mu(\emptyset)=0$;

then the function

$$
h(x)=\int_{q<|a| \leqslant 1} K(x, a) d \mu(a)
$$

belongs to $\mathcal{H}_{H}$ and vice versa each $h \in \mathcal{H}_{H}$ which admits a $\delta$-subharmonic continuation on $\stackrel{\circ}{\mathbb{R}}^{3}$ satisfies the representation

$$
h(x)=C+\int_{q<|a| \leqslant 1} K(x, a) d \mu(a),
$$

where $C$ is a constant and $\mu$ satisfies 1)-3).

Proof. Let $\mu$ satisfy Conditions 1), 2). According to Theorem B, function $h$ defined by (5) is multiplicatively periodic $\delta$-subharmonic in $\stackrel{\circ}{\mathbb{R}}^{3}$ of multiplicator $q$. In virtue of condition 3$) h$ is harmonic in $\overrightarrow{\mathbb{R}}^{3}$. Taking into account that $K(x, a)$ is independent of $A$ we have $h \in \mathcal{H}_{H}$.

Now let $h$ be a function from $\mathcal{H}_{H}$ admitting a $\delta$-subharmonic continuation on $\stackrel{\circ}{\mathbb{R}}^{3}$. According to Theorems A and B it has representation (6), where $\mu$ satisfies Conditions 1), 2). Since $\triangle h=0$ in $\overrightarrow{\mathbb{R}}^{3}, \mu$ satisfies also Condition 3). This completes the proof.

\section{DOUBLE PERIODIC HARMONIC FUNCTIONS IN A LAYER}

Let $h(x)$ be a multiplicatively periodic harmonic in $\overrightarrow{\mathbb{R}}^{3}$ function of multiplicator $q, 0<q \neq 1$. Consider the mapping

$$
x_{1}=e^{\xi} \cos \eta, \quad x_{2}=e^{\xi} \sin \eta, \quad x_{3}=e^{\xi} \cot \zeta,
$$

where $\xi, \eta \in \mathbb{R}, 0<\zeta<\pi$. We have $x_{1}^{2}+x_{2}^{2}=e^{2 \xi}>0$. Hence, it maps the layer $\{(\xi, \eta, \zeta)$ : $\xi, \eta \in \mathbb{R}, 0<\zeta<\pi\}$ onto $\overrightarrow{\mathbb{R}}^{3}$ with the Jacobians $\mathcal{J}=\frac{-e^{3 \xi}}{\sin ^{2} \zeta}$. Laplacian $\triangle$ becomes

$$
\triangle=e^{-2 \xi}\left(\frac{\partial^{2}}{\partial \xi^{2}}+\frac{\partial^{2}}{\partial \eta^{2}}+\sin ^{2} \zeta \frac{\partial^{2}}{\partial \zeta^{2}}+\sin 2 \zeta\left(\frac{1}{2} \frac{\partial}{\partial \zeta}+\frac{\partial^{2}}{\partial \xi \partial \zeta}\right)\right) .
$$

Denote

$$
g(\xi, \eta, \zeta)=h\left(e^{\xi} \cos \eta, e^{\xi} \sin \eta, e^{\xi} \cot \zeta\right) .
$$

The function $g$ is defined in the layer $\{(\xi, \eta, \zeta): \xi, \eta \in \mathbb{R}, 0<\zeta<\pi\}$.

Since $h(q x)=h(x)$, we have

$$
g(\xi+\log q, \eta+2 \pi, \zeta)=g(\xi, \eta, \zeta) .
$$

Indeed,

$$
\begin{gathered}
g(\xi+\log q, \eta+2 \pi, \zeta)=h\left(e^{\xi+\log q} \cos (\eta+2 \pi), e^{\xi+\log q} \sin (\eta+2 \pi), e^{\xi+\log q} \cot \zeta\right)= \\
\quad=h\left(q e^{\xi} \cos \eta, q e^{\xi} \sin \eta, q e^{\xi} \cot \zeta\right)=h\left(e^{\xi} \cos \eta, e^{\xi} \sin \eta, e^{\xi} \cot \zeta\right)=g(\xi, \eta, \zeta) .
\end{gathered}
$$

Denoting $\omega_{1}=\log q, \omega_{2}=2 \pi$, we obtain double periodic harmonic function $g$ of period $\Lambda=\left(\mathbb{Z} \omega_{1}, \mathbb{Z} \omega_{2}, \zeta\right)$. That is, such a function is stationary with respect to a group of the translations indicated above. 
Remark. The connection between the local spherical coordinates and the new substitution is as follows

$$
e^{\xi}=r \sin \theta, \quad \eta=\phi, \quad \zeta=\theta
$$

\section{REFERENCES}

1. O. Rausenberger Lehrbuch der Theorie der Periodischen Functionen Einer variabeln. Leipzig, Druck und Ferlag von B.G.Teubner, 1884, 470p.

2. G. Valiron Cours d'Analyse Mathematique, Theorie des fonctions, 3nd Edition. Masson et. Cie., Paris, 1966, 522 pp.

3. Y. Hellegouarch Invitation to the Mathematics of Fermat-Wiles. Academic Press, 2002, 381 pp.

4. B.A. Rozenfeld Noneuclidean spaces. Nauka, Moscow, 1969, 548p.(in Russian)

5. E.M. Stein, G. Weiss Introduction to Fourier analysis on Euclidean spaces. Princeton, New Jersey, Princeton University Press, 1974, 332p. (in Russian)

6. A.A. Kondratyuk, V.S. Zaborovska Multiplicatively periodic subharmonic functions in the punctured Euclidean space // Mat. Stud. 40 (2013). P. 159-164.

7. V.S. Khoroshchak, A.A. Kondratyuk The Riesz measures and a representation of multiplicatively periodic $\delta$-subharmonic functions in a punctured Euclidean space// Mat. Stud., 43 (2015). P. 61-65.

Vasylyna Stepanivna Khoroshchak,

Ivan Franko National University of Lviv,

1, Universytetska St.,

79000, Lviv, Ukraine

E-mail: vasylyna1992@rambler.ru

Andriy Andriyovych Kondratyuk,

Ivan Franko National University of Lviv,

1, Universytetska St.,

79000, Lviv, Ukraine

E-mail: kond@franko.lviv.ua 